\title{
Accelerated induction of etorphine immobilization in blue wildebeest (Connochaetes taurinus) through the addition of hyaluronidase
}

\author{
Mark J Dittberner ${ }^{1},{ }^{2}$, Leon $\operatorname{Venter}^{1} \&$ Vinny Naidoo ${ }^{1,3}$ \\ ${ }^{1}$ Department of Paraclinical Sciences, Faculty of Veterinary Science, University of Pretoria, Pretoria, South Africa \\ ${ }^{2}$ Department of Production Animal Studies, Faculty of Veterinary Science, University of Pretoria, Pretoria, South Africa \\ ${ }^{3}$ Biomedical Research Centre, Faculty of Veterinary Science, University of Pretoria, Pretoria, South Africa
}

Correspondence: Vinny Naidoo, Faculty of Veterinary Science, Biomedical Research Centre, University of Pretoria, Private Bag X04, Onderstepoort 0110, South Africa. E-mail: vinny.naidoo@up.ac.za

\begin{abstract}
Objective To study the effects of the addition of hyaluronidase (HA) to an etorphine/azaperone drug combination on induction times of immobilization.
\end{abstract}

Study design Experimental part-randomized 'blinded' cross-over study.

Animals Eight wild managed blue wildebeest (Connochaetes taurinus).

Methods Animals were immobilized, on separate occasions separated by two weeks, with one of four treatments. Treatments were; 'Control drugs (CD), etorphine $0.01 \mathrm{mg} \mathrm{kg}{ }^{-1}+$ azaperone at $0.1 \mathrm{mg} \mathrm{kg}{ }^{-1}$; treatment $1 \mathrm{CD}+5000 \mathrm{IU} \mathrm{HA}$; treatment $2 \mathrm{CD}+7500$ IU HA; and treatment 3 etorphine $0.007 \mathrm{mg} \mathrm{kg}^{-1}+$ azaperone at $0.07 \mathrm{mg} \mathrm{kg}^{-1}+7500$ IU HA. Times to first effect and to immobilization (from darting to possible to approach and blindfold) were measured. ANovA was used to compare treatments. Results are given in means $\pm \mathrm{SD}$ (range).

Results For control, and treatments 1-3 respectively, times (in minutes) to first effect were $1.58 \pm 0.42 \quad(1.02-2.10), \quad 1.64 \pm 0.42 \quad(0.95-$ $2.17), 1.12 \pm 0.24(0.80-1.48)$ and $1.60 \pm 0.21$ (1.13-1.88) and to immobilization were
$5.38 \pm 1.53 \quad(3.82-8.07), \quad 3.80 \pm 1.14 \quad(2.02-$ $5.50), 3.51 \pm 1.08(2.28-5.52)$ and $4.46 \pm 0.67$ (3.30-5.40). Compared to control, time to first effect for treatment 2 was significantly shorter. Time to immobilization was significantly quicker in all three treatments containing HA than that for control.

Conclusion and clinical relevance Hyaluronidase can reduce the time to immobilization when used in the immobilizing dart, and might be usefully incorporated into etorphine combinations for darting wildlife.

Keywords azaperone, blue wildebeest, etorphine, hyaluronidase, immobilization time.

\section{Introduction}

Free roaming wild animals usually are chemically immobilized for translocation, treatment, testing/ sampling or for the fitting of tracking devices. During this immobilization, it is important to ensure that the time to induction of anaesthesia is kept as short as possible to avoid complications such as injuries, hyperthermia and capture myopathy. Since no single drug provides this rapid induction of immobilization, combination protocols with sedatives/ tranquilizers are employed, taking advantage of the combination anaesthetic effect (Haigh 1990). While the use of drug combination without doubt is 
effective, further improvements of induction times would be preferable. One method suggested to facilitate this quicker onset could be the inclusion of hyaluronidase (HA) into the drug mixture.

The HA protein initially was demonstrated to play an important role in enabling sperm penetration of the cumulus cells surrounding the mammalian egg. The enzyme was later shown to be responsible for the spread of the venom of bees and wasps wherein it is also present. The compound has been associated with various bacterial strains, by assisting with tissue spread. While the mechanism of action of HA is not completely understood, it is believed that it is responsible for the cleavage of the interstitial cellular barrier through the disruption of the $\beta-1,4$ glycosidic bond between 2-acetamido-2-deoxy-D-glucose and D-glucuronic acid in a manner which is quickly reversible (Watson 1993).

Based on the ability of the HA protein to enhance the spread of natural toxins and/or infectious agents, it has long been speculated that the product could be beneficial in aiding in drug absorption. This is largely based on Fick's law, which relates the rate of diffusion across a membrane to the surface area over which said diffusion results. As such if hylauronidase can temporarily interfere with the intercellular barriers, it will increase the surface area of diffusion and thus absorption of the drug.

HA has been utilized in combination with local anaesthetic agents (Kallio et al. 2000) and also in a number of wildlife dart combinations. It was found that immobilized Cuban crocodiles (Crocodylus rhombifer) recovered significantly sooner when HA was included in the antidote (Lloyd et al. 1994). During a moose capture, the addition of HA to a fentanyl/ xylazine mixture appeared to decrease immobilization times by between 36 and 45\% (although not confirmed statistically) (Haigh et al. 1977). In addition to speeding up immobilisation times, studies in polar bears have also suggested that the addition of HA to combinations reduces the amount of immobilizing agents required (Cattet \& Obbard 2010). Unfortunately none of these studies were designed in a sufficiently robust manner to conclusively prove the benefit of presence of the HA in the dart mixture as they relied on the use of parallel study designs.

The aim of this study was to quantify the effect, in blue wildebeest (Connochaetes taurinus), of two doses of HA on etorphine/azaperone in the immobilization, and also the effect of the addition of one dose of HA on two doses of etorphine/azaperone using a crossover study design.

\section{Materials and methods}

\section{Study design}

This study was approved by the animal use and care committee of the University of Pretoria (V017-09). Eight blue wildebeest (four of each sex, between 1.5 and 5.5 years) were used. The wildebeest were mass captured in Hoedspruit (Mpumalanga, South Africa) and transported to the study enclosures in Malelane (Mpumalanga, South Africa) prior to a four week adaptation period. Animals received a diet of dried Erogrottis teff, Lucerne (Medicago sativa) and game pellets (Epol, South Africa) and had ad lib access to pumped ground water. Two weeks prior to the study initiation, each animal was immobilized with etorphine (2-3 mg) and azaperone (20-30 mg) for weighing and the placement of ear tags.

The study made use of a four-way cross-over study design, with four treatments, four sequences and four periods. Observers were unaware of which treatment had been used. A wash-out period of two weeks was allowed between treatments, this period being based on field experience which indicated no differences in subsequent response times in immobilization after this inter-capture interval. The treatments were

- Control drugs (CD). These were the standard dose of immobilizing drugs (IDs)- etorphine (0.01 $\mathrm{mg} \mathrm{kg}^{-1}$ ) (Captivon, Wildlife Pharmaceuticals, South Africa) and azaperone $\left(0.1 \mathrm{mg} \mathrm{kg}^{-1}\right)$ (Stresnil, Janssen Pharmaceuticals, South Africa) as per manufacturers recommendation.

- Treatment 1. CD + Low dose HA. This consisted of the control drugs plus 5000 IU HA (Hyalase, Merck, South Africa)

- Treatment 2. CD + high dose HA. This consisted of the control drugs plus 7500 IU HA.

- Treatment 3. (Low dose CD + high dose HA). This consisted of $75 \%$ of the original control doses of etorphine and of azperone, together with 7500 IUHA.

All darts were brought up to the same volume by addition of sterile water prior to administration per phase. All treatments were administered into the hindquarters by $3 \mathrm{~mL}$ Dan-Inject darts, propelled from a Dan-Inject (Fritz Rohr, Skukuza) dart gun.

Prior to the start of the study, the animals were allocated part-randomly to treatment so that each period always had two animals per treatment group. For every subsequent phase the treatments were changed such that all animals had received the four treatments by the study end. Animals were immobi- 
lized in the morning, when ambient temperatures ranged from 18 to $36{ }^{\circ} \mathrm{C}$. Following dart removal, penetration wounds were treated with $5 \mathrm{~mL}$ procaine penicillin (Lentrax, Merial, South Africa) administered directly into the wound. Immobilizations were reversed with naltrexone (Trexonil 5\% m/v; Wildlife Pharmaceuticals, South Africa) administered intravenously in an ear vein at $0.2 \mathrm{mg} \mathrm{kg}^{-1}$.

\section{Monitoring of anaesthesia}

Animals were observed for time taken for the first indication of sedation (reported as first effect) e.g. subtle changes in behaviour such as slight imbalance or a stumble. The time to immobilization was recorded as the time taken from the darting to when animal could be approached safely and blindfolded. All monitoring was undertaken by the same veterinarian who has substantial experience in the immobilization and monitoring of wildlife. The said veterinarian was unaware to the administered treatments.

\section{Statistics}

All results are presented as means \pm standard deviation (range). On demonstration of normality using a Shapiro-Wilk's test, differences were deter- mined using a univariate ANOVA with sequence, period, treatment, subject and sex as factors. Posthoc analysis made use of a Dunnett's $t$-test for effects for parameters being lower than the control group. All analysis was undertaken in SPSS, version 20.0 (SPSS, IL, USA).

\section{Results}

Table 1 gives the times to first effect and to immobilization with the four treatments. The time to first effect for treatment 2, CD + high dose HA was significantly faster than that for the control treatment $(p=0.02)$. Time to immobilization was significantly faster with all HA treatments than the control that had no HA. When compared with the control, significances were, for treatment 3, low dose $\mathrm{CD}+$ high doses $\mathrm{HD}$, $p=0.045$; for treatment $2 \mathrm{CD}+$ high dose $\mathrm{HA}$ $p=0.006$, and for treatment $1 \mathrm{CD}+$ low dose HA $p=0.008$. Once immobilized the quality of the anaesthesia was comparable between the groups. No side effects were reported during the study.

\section{Discussion}

For this study we speculated that the addition of HA to the dart combination would offer no significant

Table 1 Times (in minutes) from dart penetration to stages of immobilization in eight blue wildebeests given, on separate occasions, four treatments. Results are mean \pm SD (range)

\begin{tabular}{|c|c|c|}
\hline Treatments & $\begin{array}{l}\text { Time (minutes) to } \\
\text { first signs }\end{array}$ & $\begin{array}{l}\text { Time (minutes) to } \\
\text { immobilization }\end{array}$ \\
\hline $\begin{array}{l}\text { Control } \\
\text { Control drugs (CD) } \\
\text { Etorphine } 0.01 \mathrm{mg} \mathrm{kg}^{-1} \\
\text { Azaperone } 0.1 \mathrm{mg} \mathrm{kg}^{-1}\end{array}$ & $1.58 \pm 0.42(1.02-2.10)^{\mathrm{a}}$ & $5.38 \pm 1.53(3.82-8.07)^{\mathrm{bcd}}$ \\
\hline $\begin{array}{l}\text { Treatment } 1 \\
\qquad \begin{array}{l}\text { CD + Low dose HA } \\
\text { Etorphine } 0.01 \mathrm{mg} \mathrm{kg}^{-1} \\
\text { Azaperone } 0.1 \mathrm{mg} \mathrm{kg}^{-1} \\
5000 \mathrm{IU} \mathrm{HA}\end{array}\end{array}$ & $1.64 \pm 0.42(0.95-2.17)$ & $3.80 \pm 1.14(2.02-5.50)^{c}$ \\
\hline $\begin{array}{l}\text { Treatment } 2 \\
\qquad \mathrm{CD}+\text { High dose } \mathrm{HA} \\
\text { Etorphine } 0.01 \mathrm{mg} \mathrm{kg}^{-1} \\
\text { Azaperone } 0.1 \mathrm{mg} \mathrm{kg}^{-1} \\
7500 \mathrm{IU} \mathrm{HA}\end{array}$ & $1.12 \pm 0.24(0.80-1.48)^{\mathrm{a}}$ & $3.51 \pm 1.08(2.28-5.52)^{d}$ \\
\hline $\begin{array}{l}\text { Treatment } 3 \\
\text { Low dose CD }+ \text { high dose HA } \\
\text { Etorphine } 0.007 \mathrm{mg} \mathrm{kg}^{-1} \\
\text { Azaperone } 0.07 \mathrm{mg} \mathrm{kg}^{-1} \\
7500 \text { IU HA }\end{array}$ & $1.60 \pm 0.21(1.13-1.88)$ & $4.46 \pm 0.67(3.30-5.40)^{b}$ \\
\hline
\end{tabular}

Results sharing the same superscript letter differ significantly from each other. CD, control drugs; HA, hyaluronidase. 
benefit in reducing time to immobilization in blue wildebeest. When the 5000 and 7500 IU HA treatment groups were compared to the control to which no HA was added, both treatment groups showed significantly reduced times to immobilization which in real terms amounted to 100 seconds. The benefit of this reduction in time to immobilization is that less distance is covered by the animal between darting and immobilization. We estimate that animals may achieve an approximate speed of $20 \mathrm{~km} \mathrm{hour}^{-1}$ during the excitatory phase prior to immobilization, so the 100 seconds saved during immobilization could decrease the distance covered by the animal by up to $550 \mathrm{~m}$. This reduced distance firstly would reduce the risk of capture myopathy, hyperthermia and acidosis, and secondly reduces the risk of animals being lost into thick brush (Meltzer et al. 2006).

When the dose of HA in the dart was increased from 5000 to 7500 IU (50\%), a further reduction of the time to immobilization was evident, indicating the presence of a dose response relationship although the reduction did not reach statistical significance. This finding differed to that described in a study examining the speed of onset of ophthalmic local anaesthetic nerve blocks, whereby the addition of $3.75 \mathrm{IU} \mathrm{mL}^{-1}$ and $7.5 \mathrm{IU} \mathrm{mL}^{-1} \mathrm{HA}$ to a bupivicaine-lidocaine mixture produced the same time to effect in comparison to the control (Kallio et al. 2000). The latter study concluded that no clear dose/response relationship between HA dose and quality of block existed. While the exact reason for the difference is unknown, the space within the drug is administered is probably directly related to the effect achieved. For the orbit, where the space available for diffusion and absorption is limited, further increases in doses of HA might not increase absorption as the maximum effect in the available tissue would be achieved. However the muscle of the hindquarters, as used in this study, would provide a much larger site over-which the HA could function, thereby providing the possibility of a dose response relationship.

For the low dose etorphine and azaperone treatment group with 7500 IU HA the time to immobilization was lower, and range smaller than for the control doses, indicated a potential dose sparing effect, as more rapid uptake would result in a faster achievement of the required blood concentrations for immobilization. This supports the conclusion of Cattet \& Obbard (2010) who found that the addition of HA reduced the total dose of xylazine/zolazepam/ tiletamine required for immobilisation of free-ranging polar bears. However, it is also possible that the effective concentration required for the immobilization of wildebeest is probably lower than the current recommended dose. For a better understanding of the dose sparing effect of HA, it should ideally be compared to a control with the lower dose of etorphine and azaperone in the absence of HA.

During our study none of the animal experienced pain, discomfort or swelling at the dart sites. This supported the findings of an earlier study where no sloughing or inflammation at the sites of injection in 519 cases of local and nerve block analgesia using pentocaine hydrochloride with HA were recorded (Moore 1950). One of the other concerns with the use of HA is the possibility of infection in the dead space created when the interstitial barrier between cells is broken down, especially since the dart enters through dirty hair. This study did not look at this possibility as all the animals were always treated with antibiotics.

In conclusion the inclusion of $\mathrm{HA}$ in the dart formulation increased the speed of induction of immobilization compared with a formulation of etorphine/azaperone at the standard dose without HA. There was also a tendency for a dose response relationship in the effect of HA, but this did not reach statistical significance. In addition, based on the similar time to effect for the lower dose of etorphine/ azaperone in the presence of 7500 IU HA to a standard dose in the absence of HA, indicates that the beneficial effects from HA resides in its ability to enhance absorption. Therefore the addition of HA into immobilization formulations may be useful for wildlife immobilizations using etorphine.

\section{Acknowledgements}

The authors would like to thank Dr Cobus Raath for allowing the study to take place on his farm and supplying the animals, drugs and accommodation. Financial assistance was provided by the University of Pretoria and the South African Veterinary Foundation.

\section{References}

Cattet MR, Obbard ME (2010) Use of hyaluronidase to improve chemical immobilization of free-ranging polar bears (Ursus maritimus). J Wildl Dis 46, 246-250.

Haigh JC (1990) Opioids in zoological medicine. J Zoo Wildlife Med 21, 391-413. 
Haigh JC, Stewart RR, Frokjer R et al. (1977) Capture of moose with fentanyl and xylazine. J Zoo Anim Med 8, 22-29.

Kallio H, Paloheimo M, Maunuksela EL (2000) Hyaluronidase as an adjuvant in bupivicaine-lidocaine mixture for retrobulbar/peribulbar block. Anesth Analg 91, 934-937.

Lloyd ML, Reichard T, Odum RA (1994) Gallamine reversal in Cuban crocodiles (Crocodylus rhombifer) using neostigmine alone versus neostigmine with hyaluronidase. Proceedings of the Association of Reptilian \& Amphibian Veterinarians and American Association of Zoo Veterinarians, Pittsburgh, USA, pp. 117-120.
Meltzer D, Burroughs R, Morkel P (2006) Applied pharmacology. In: Chemical and Physical Restraint of Wild Animals (1st edn). Mckenzie AA (ed.). Published jointly by the Wildlife Division Support Services CC and the South African Veterinary Foundation, Greyton, South Africa. pp. 43-67.

Moore DC (1950) An evaluation of hyaluronidase in local and nerve block analgesia: a review of 519 cases. Anesthesiology 11, 470-484.

Watson D (1993) Hyaluronidase. Brit J Anaesth 71, 422425. 